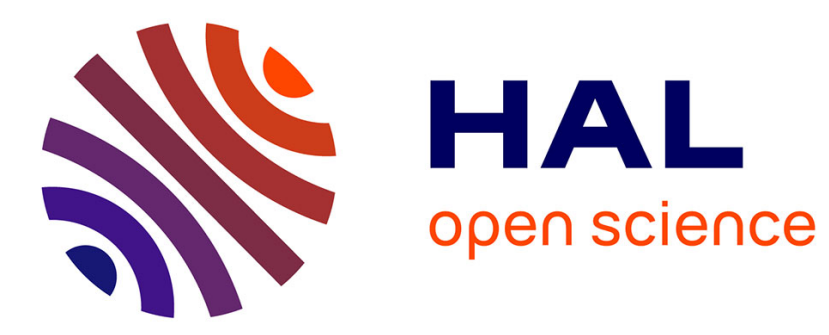

\title{
An Equivalent F-Number for Light Field Systems: Light Efficiency, Signal-to-Noise Ratio, and Depth of Field Ivo Ihrke
}

\section{To cite this version:}

Ivo Ihrke. An Equivalent F-Number for Light Field Systems: Light Efficiency, Signal-to-Noise Ratio, and Depth of Field. QCAV, 2019, Mulhouse, France. hal-02144991

\author{
HAL Id: hal-02144991 \\ https://hal.inria.fr/hal-02144991
}

Submitted on 3 Jun 2019

HAL is a multi-disciplinary open access archive for the deposit and dissemination of scientific research documents, whether they are published or not. The documents may come from teaching and research institutions in France or abroad, or from public or private research centers.
L'archive ouverte pluridisciplinaire HAL, est destinée au dépôt et à la diffusion de documents scientifiques de niveau recherche, publiés ou non, émanant des établissements d'enseignement et de recherche français ou étrangers, des laboratoires publics ou privés. 


\title{
An Equivalent F-Number for Light Field Systems: Light Efficiency, Signal-to-Noise Ratio, and Depth of Field
}

\author{
Ivo Ihrke \\ independent scientist
}

\begin{abstract}
The paper discusses the light efficiency and SNR of light field imaging systems in comparison to classical 2D imaging. In order to achieve this goal, I define the "equivalent f-number" as a concept of capturing the light gathering ability of the light field system. Since the f-number, in classical imaging systems, is conceptually also linked with the depth-of-field, I discuss an appropriate depth-of-field interpretation for light field systems.
\end{abstract}

Keywords: light field imaging, light efficiency, f-number, equivalent f-number

\section{INTRODUCTION}

Even though the $\mathrm{F} / \#$ is a simple concept, it captures major important features of imaging systems and is therefore in popular use. The F/\# describes the light efficiency of an optical system as well as the corresponding loss in depth-of-field for higher performing lenses (smaller $\mathrm{F} / \#$ ).

To my knowledge, so far, the concept has not been applied to light field imaging systems. It is therefore my goal in the current article to discuss the major features of light efficency, SNR, and depth-of-field and their implications when comparing standard 2D imaging systems with light field imaging systems: Ultimately, the users of such systems need intuitive concepts for evaluating and comparing different systems.

After introducing some background that mostly serves to introduce the notation used in this article, Sect. 2, I proceed in analyzing the light efficiency and the resulting SNR in Sect. 3. This analysis is performed in a simplified 2D setting in order to remove unnecessary technical complexity from the formulas. A rough extension to $3 \mathrm{D}$ systems is then proposed in Sect. 4, which also introduces the definition of the equivalent $\mathrm{F} / \#$ for light field systems. Finally, the scaling laws for the depth-of-field are investigated in Sect. 5, where it is seen that the concept relates to the virtually achievable DoF in synthesized light field images.

\section{BACKGROUND}

Light efficiency is an important consideration in optical design and, consequently, several concepts are in use: the (image-side) f-number of an optical system describes the opening angle of the cone of light incident on the sensor after it has passed the optical system, assuming an object point at infinity. When the object point is closer to the lens, usually, the concept of working f-number is employed. The extent of the cone of light passed into the optical system or emerging from it into a focused spot on the sensor are known as the object-side and image-side numerical aperture (NA), respectively. The NA is defined as $\mathrm{NA}=n \sin (\alpha)$, with $\alpha$ being the half-angle of the extent of the light cone and $n$ the refractive index, Fig. 1.

The working f-number is related to the image-space numerical aperture by

$$
\mathrm{F} / \#_{w}=\frac{1}{2 \mathrm{NA}_{i}},
$$

and the object-space numerical aperture is given in terms of the optical system's magnification $M=h_{i} / h_{o}$ by

$$
\mathrm{NA}_{o}=M \cdot \mathrm{NA}_{i}
$$

where $h_{i}$ and $h_{o}$ are an image and object space height, respectively. In the following, I will use $h_{i}$ and $h_{o}$ to indicate image and object-space pixel sizes, respectively.

Pixel values are proportional to the radiant energy received by them*. Abstracting from the adjustable

Further author information: (Send correspondence to I.I.)

I.I: E-mail: ivo.ihrke@inria.fr

*aside from non-linear transfer curves of the sensor 
exposure time, the radiant flux is the interesting quantity to evaluate light efficiency.

The étendue $G$ of an optical system is a measure proportional to the radiant flux passing through some area and solid angle: ${ }^{1}$

$$
G=h_{i} \cdot \mathrm{NA}_{i}=h_{o} \cdot \mathrm{NA}_{o}
$$

where the equality on the right side is due to Liouville's theorem ${ }^{\dagger}$. The area in question is the pixel area and the solid angle is the bundle of light passed by the optical system onto the sensor.

\section{AN OBJECT-SPACE SIMPLIFIED MODEL OF LIGHT FIELD IMAGING}

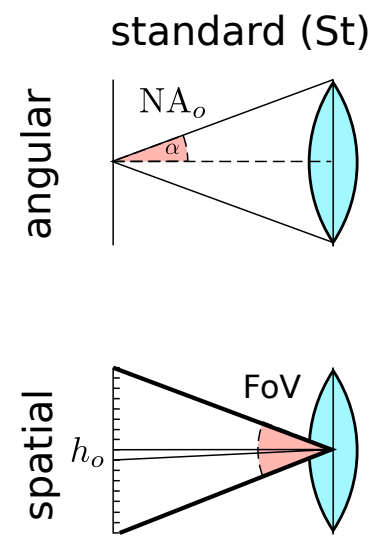

a) light field (LF)
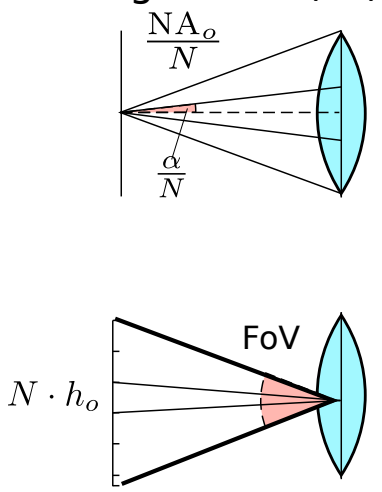

b) low-resolved (LR)
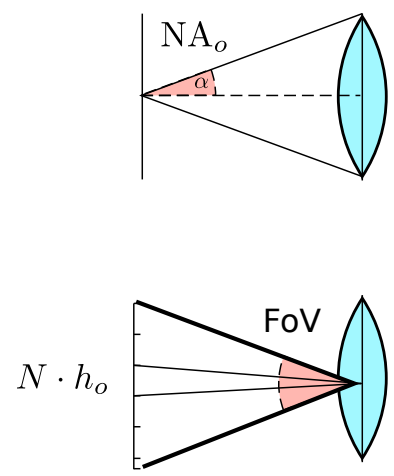

c)

Figure 1. Object-side view of three comparison systems observing the same field of view. The systems a) and b) use the same hypothetical sensor, system c) uses a sensor of same size but with larger pixels. Left: standard imaging Middle: light field imaging. Right: standard imaging with a low-resolution sensor (same physical size as in the other cases). In the case of the light field system, the aperture is subdivided by a factor of, in this case $N=3$, the object-side pixel size $h_{o}$ of the standard system is increased by the same factor. The low-resolution system has the full NA of the standard system, but a smaller number of larger pixels chosen such as to match those of a light field subview.

I will use Fig. 1 to perform a comparative evaluation between standard imaging a), light field imaging b), and a low-resolution standard imaging system c) in terms of their light efficiency. For this, I will use a 2D example to simplify the exposition, however, the development is straightforward to generalize to 3D and the results are similar. I will further assume that the light field system subdivides the aperture by a factor of $N$, i.e. that $N$ subviews are generated by it. The analysis is, in the spirit of Mignard-Debise et al., ${ }^{2}$ performed on the object-side of the imaging system only and may, for the light field system, refer to a virtual camera system. This way, the analysis can be performed without reference to a particular optical light field imaging implementation.

\subsection{Light Efficiency with the Same Sensor}

Assume the setting in Fig. 1 a) and b), i.e. a standard imaging system and a light field imaging system, both constructed using the same sensor and with the same field of view. The light field system produces $N$ subviews as shown in b). Since the field of view is constant, the resolution is decreased by a factor of $N$, i.e. the object-side pixel size $h_{o}^{\mathrm{LF}}=N \cdot h_{o}^{\mathrm{St}}$ is increased by the same factor. Here, and in the subsequent, the upper indices "LF" and "St" refer to light field and standard imaging properties, respectively.

At the same time, the object-side NA of the light field subviews $\mathrm{NA}_{o}^{\mathrm{LF}}$ is decreased approximately by a factor of $N$ as compared to the standard system:

$$
\mathrm{NA}_{o}^{\mathrm{LF}}=\sin \frac{\alpha^{\mathrm{St}}}{N} \approx \frac{\mathrm{NA}_{o}^{\mathrm{St}}}{N}
$$

\footnotetext{
${ }^{\dagger}$ For simplicity of argument, I am defining here a one-dimensional version of étendue, properly speaking, $h_{i}, h_{o}$ should be two-dimensional areas and $\mathrm{NA}_{i}, \mathrm{NA}_{o}$ should be replaced by solid angles.
} 
where $\alpha^{\mathrm{St}}$ is the opening half-angle of the cone of light admitted into the standard imaging system from the object point and the medium is air. Using Eq. (3), this implies

$$
G^{\mathrm{LF}}=h_{o}^{\mathrm{LF}} \cdot \mathrm{NA}_{o}^{\mathrm{LF}}=N \cdot h_{o}^{\mathrm{St}} \cdot \frac{\mathrm{NA}_{o}^{\mathrm{St}}}{N}=h_{o}^{\mathrm{St}} \cdot \mathrm{NA}_{o}^{\mathrm{St}}=G^{\mathrm{St}},
$$

i.e. the étendue, and therefore the light gathered by a pixel, is the same in both cases: a standard system pixel and a light field system pixel receive the same exposure. This also implies that their SNR is the same.

\subsection{Light Efficiency with an Equivalently Low-Resolved Sensor}

The light field subviews have an $N \times$ lower resolution while using the same overall sensor area as the standard comparison system. It therefore seems plausible to compare against a low-resolved sensor of similar size (superscript LR), Fig. 1 c). In this case, the object-side pixel size is the same as in the case of the light field subview, i.e. $h_{o}^{\mathrm{LR}}=N \cdot h_{o}^{\mathrm{St}}$ and the $\mathrm{NA}$ is the same as in standard imaging $\mathrm{NA}_{o}^{\mathrm{LR}}=\mathrm{NA}_{o}^{\mathrm{St}}$, i.e. the étendue is

$$
G^{\mathrm{LR}}=N \cdot h_{o}^{\mathrm{St}} \cdot \mathrm{NA}_{o}^{\mathrm{St}}=N \cdot G^{\mathrm{St}},
$$

i.e. $N \times$ increased as compared to the light field subview.

\subsection{Signal-to-Noise Ratio}

To assess the impact on the signal-to-noise ratio (SNR), I introduce a simple noise model:

$$
\sigma_{\text {tot }}^{2}=\sigma_{\mathrm{r}}^{2}+\sigma_{\mathrm{p}}^{2}
$$

i.e. a combination of read noise $\sigma_{\mathrm{r}}$ and photon noise $\sigma_{\mathrm{p}}$ for a pixel. The read noise depends on the operating conditions and electronics of the pixel (but not its size) and can be assumed to be equal for the comparison systems $^{\ddagger}$, whereas the photon noise is inherent in measuring the arriving photons and thus depends on the étendue. The photon noise is proportional to the square root of the signal, i.e.

$$
\sigma_{\mathrm{p}} \propto \sqrt{G} .
$$

The SNR is the ratio of signal over noise:

$$
\mathrm{SNR}=\frac{G}{\sigma_{\mathrm{tot}}}=\frac{h_{i}}{2 \mathrm{~F} / \#_{w} \sigma_{\mathrm{tot}}},
$$

where the last equality is due to combining Eqs. (1) and (3). Returning to the comparison with the low-resolved comparison system (LR), the effect in terms of signal-to-noise ratio (SNR) of a single pixel is an approximate $\sqrt{N}$ improvement of the low-resolved standard system over the pixels in the individual light field subviews and the pixels in the high-resolved standard system:

$$
\begin{aligned}
& \mathrm{SNR}^{\mathrm{LR}} \stackrel{(9)}{=} \frac{G^{\mathrm{LR}}}{\sigma_{\mathrm{tot}}^{\mathrm{LR}}} \\
& \stackrel{(6),(7)}{=} \frac{N \cdot G^{\mathrm{St}}}{\sqrt{\sigma_{\mathrm{p}}^{2 \mathrm{LR}}+\sigma_{\mathrm{r}}^{2 \mathrm{LR}}}} \\
& \stackrel{(6),(8)}{=} \frac{N \cdot G^{\mathrm{St}}}{\sqrt{N \cdot \sigma_{\mathrm{p}}^{2 \mathrm{St}}+\sigma_{\mathrm{r}}^{2 \mathrm{St}}}}
\end{aligned}
$$

where I assume that the read noise is the same for both cases, i.e. $\sigma_{\mathrm{r}}^{\mathrm{LR}}=\sigma_{\mathrm{r}}^{\mathrm{St}}$.

\footnotetext{
${ }^{\ddagger}$ Dark noise could be added and modelled as depending on the pixel size, assuming that the quantum well scales with the size of the pixel.
} 
Case I: negligible read noise: If read noise is negligible, i.e. photon noise dominates over other noise sources such as the read noise $\sigma_{\mathrm{r}} \ll \sigma_{\mathrm{p}}$, the expression may be further simplified to

$$
\mathrm{SNR}^{\mathrm{LR}}=\sqrt{N} \cdot \frac{G^{\mathrm{St}}}{\sigma_{\text {tot }}^{\mathrm{St}}}=\sqrt{N} \cdot \mathrm{SNR}^{\mathrm{St}}=\sqrt{N} \cdot \mathrm{SNR}_{\text {oneview }}^{\mathrm{LF}},
$$

where the last equality is due to the same étendue for the light field (LF) and the high-resolution standard (St) system, Eq. (5). An important observation is that this argument holds for each of the $N$ individual subviews of the light field system (LF), whereas the low-resolved comparison system (LR) only has a single view.

Let me assume that the $N$ light field subviews can be registered (i.e. their disparity can be computed and compensated for). In this case, the registered subviews may be added digitally to produce an improved SNR. Are physical integration and digital summation equivalent?

The signal is then composed of the sum of $N$ digitally registered pixels and the SNR for negligible read noise becomes:

$$
\mathrm{SNR}^{\mathrm{LF}}=\frac{\sum_{i=1}^{N} G^{\mathrm{LF}}}{\sqrt{\sum_{i=1}^{N} \sigma_{\mathrm{p}}^{2} \mathrm{LF}}}=\sqrt{N} \cdot \mathrm{SNR}_{\text {oneview }}^{\mathrm{LF}}
$$

Comparing with Eq. (11), we see that digital averaging has, as expected, the same effect as physical integration ${ }^{\S}$, i.e. the signal-to-noise ratio of a digitally summed registered light field view is equivalent to the low-resolution comparison system (LR).

Case II: low light conditions: In low light conditions, the read noise is non-negligible. The expected difference is that, in the case of light field imaging (LF), the read noise of $N$ pixel amplifiers poses a disadvantage, as compared to the low-resolved standard imaging system (LR) where only one amplifier is present. Indeed

$$
\mathrm{SNR}^{\mathrm{LF}}=\frac{\sum_{i=1}^{N} G^{\mathrm{LF}}}{\sqrt{\sum_{i=1}^{N} \sigma_{\text {tot }}^{2} \mathrm{LF}}}=\frac{N \cdot G^{\mathrm{LF}}}{\sqrt{N \cdot\left({\sigma_{\mathrm{p}}^{2}}^{\mathrm{LF}}+{\sigma_{\mathrm{r}}^{2}}^{\mathrm{LF}}\right)}}=\frac{N \cdot G^{\mathrm{St}}}{\sqrt{N \cdot \sigma_{\mathrm{p}}^{2} \mathrm{St}+N \cdot \sigma_{\mathrm{r}}^{2} \mathrm{St}}},
$$

where the last equality is due to the equality of signals between standard imaging (St) and light field imaging (LF), Eq. (5). Comparison with Eq. (10) shows, as expected, that in low light conditions physical integration is advantageous and light field systems have a lower SNR even with digital averaging of the registered subviews.

Note that all SNR expressions can equivalently be given in terms of the working f-number F/\# ${ }_{w}$, Eq. (9).

\section{VIGNETTING IN REAL LIGHT FIELD SYSTEMS AND THE EQUIVALENT F-NUMBER}

The previous analysis assumes an idealized setting in that it is tacitly assumed that the complete light cone of the standard system can be partitioned, i.e. sub-divided without loss of rays, and that all sensor pixel can be utilized. In general, this is not the case. ${ }^{1,3}$

Real light field systems exhibit vignetting and Mignard-Debise and colleague ${ }^{3}$ introduced a separation into spatial and angular vignetting, as illustrated in Fig. 2. The illustrating example systems are kaleidoscopic light field imaging ${ }^{4}$ and lenslet-based light field imaging ${ }^{5-7}$ systems.

According to Mignard-Debise, ${ }^{3}$ vignetting is described as a scalar factor

$$
c_{\text {system }}[\%]=c_{\text {spatial }}[\%] \times c_{\text {angular }}[\%],
$$

\footnotetext{
$\S$ The presence of dark noise would not change this situation since it was assumed to scale with the pixel size.
} 

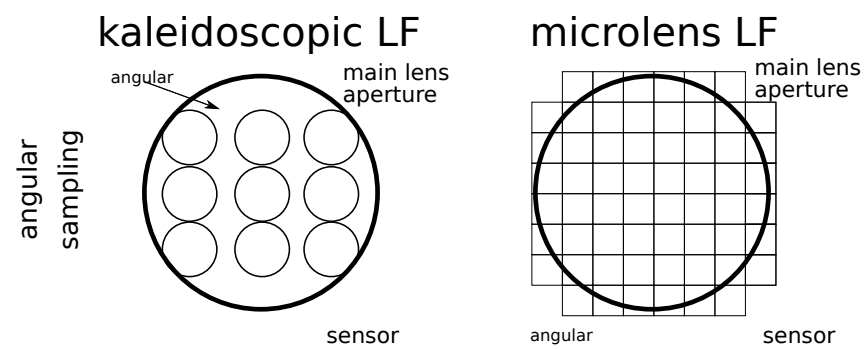

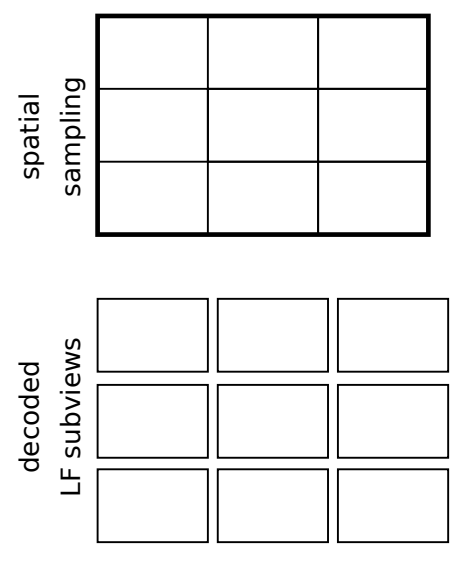

a)

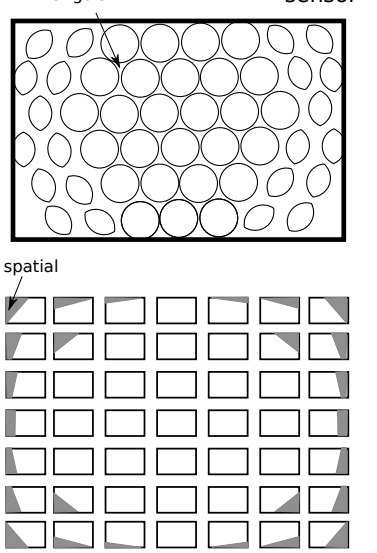

b)

Figure 2. Vignetting in two optical light field configurations Left: kaleidoscopic light field imaging, Right: lenslet-based light field imaging. The top row shows the angular sampling pattern in the aperture of the main lens of the light field system. The middle row shows the spatial sampling pattern on the sensor, while the bottom row illustrates decoded light field subviews. The areas of the main lens aperture and the sensor, respectively, that are not covered by the light field system introduce loss of light as compared to the standard 2D imaging system of Fig. 1 a). The kaleidoscopic system suffers from angular vignetting since the main lens aperture is not fully covered. The lenslet system has angular vignetting between the microlens images (middle row), but often also shows spatial vignetting (bottom row) that is introduced by the cat's-eye shape of the outer microlens images.

that multiplies the étendue of the ideal system. However, the F/\# is predominantly a measure of exposure, i.e. it applies to pixels individually. It is therefore more adequate to only consider the angular vignetting part in the definition of an equivalent étendue:

$$
\hat{G}=c_{\text {angular }} \cdot G \text {. }
$$

For a standard system, $c_{\text {angular }}=1$, i.e. it uses the full aperture. Using Eqs. (1)-(3), an equivalent working $F / \#$ can then be defined as

$$
\mathrm{F} / \#_{w}=\frac{h_{i}}{2 \cdot c_{\text {angular }} \cdot G}=M \cdot \frac{1}{2 \cdot c_{\text {angular }} \cdot \mathrm{NA}_{o}}
$$

Expressing the equivalent working F/\# of the light field system (LF) in terms of the (St) system,

$$
\mathrm{F} / \#_{w}^{\mathrm{LF}}=M^{\mathrm{LF}} \cdot \frac{1}{2 \cdot c_{\text {angular }} \cdot \mathrm{NA}_{o}^{\mathrm{LF}}}=\frac{1}{N} M^{\mathrm{St}} \cdot \frac{N}{2 \cdot c_{\text {angular }} \cdot \mathrm{NA}_{o}^{\mathrm{St}}}=\frac{1}{c_{\text {angular }}} \mathrm{F} / \#_{w}^{\mathrm{St}},
$$

it is seen that the angular vignetting factor inversely scales the working $\mathrm{F} / \#$ of the standard system. Since $0 \leq c_{\text {angular }} \leq 1$, the equivalent working F/\# can only increase. Since the working F/\# is an extension of the standard F/\#, I propose to use relation (17) also for the standard F/\# of a light field system.

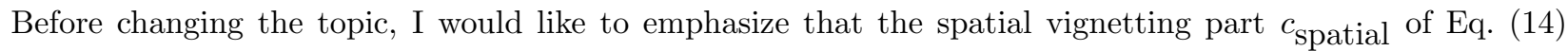
should not be forgotten in the analysis of a light field imaging system. Effectively, spatial vignetting reduces the number of "mega-rays" that can be successfully acquired with a light field imaging system. 


\section{DEPTH-OF-FIELD}

In photography, the $\mathrm{F} / \#$ not only indicates light efficiency, but, since it is describing the extent of the light cone scattered by an object point into the optical system, also the depth-of-field (DoF) properties of the system. A discussion is therefore not complete without investigating the DoF, and in particular, the scaling laws for aperture subdivision by a factor of $N$ as in light field imaging.

In order to put the discussion on a sound basis, I am sketching the derivation of the depth-of-field formula that I will use in the following. The relevant geometry is shown in Fig. 3. The argument, again, follows a predominantly object-space approach in order to abstract from particular systems. However, since the DoF is typically defined in terms of circles of confusion $(\mathrm{CoC})$ that relate to the pixel size of the sensor (e.g. the $\mathrm{CoC}$ is 1 pixel), it will be necessary to relate to the image space pixel size $h_{i}$ via the (effective) focal length of the imaging system. For light field systems that employ a main lens, the effective focal length is typically $f / N$, where $f$ is the focal length of the main lens .

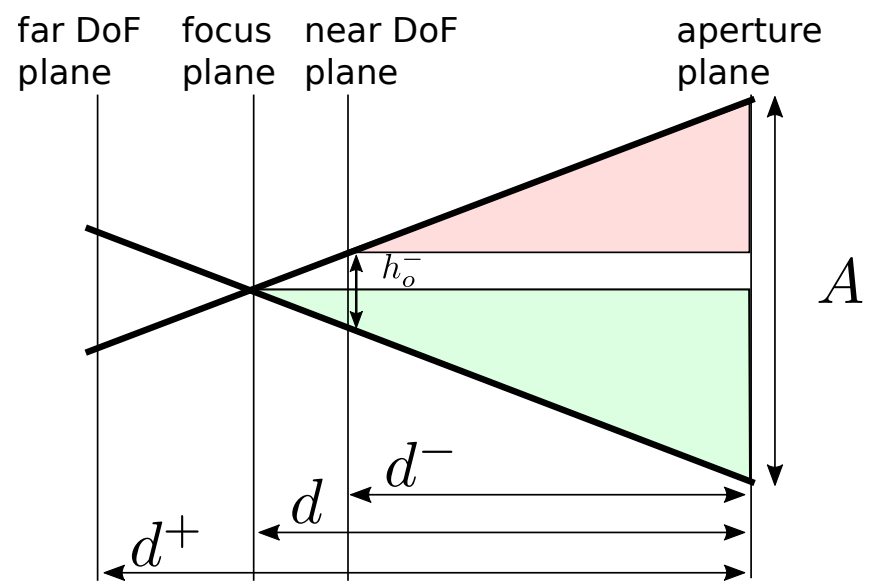

Figure 3. Sketch illustrating the geometry for the DoF discussion. $A$ is the aperture diameter, $d$ the distance of the focal plane from the aperture plane (considered positive towards the object space) and $h_{o}^{-}$the object space pixel size at the near depth-of-field plane. Similarly, $d^{-}$and $d^{+}$are distances from the aperture plane.

\subsection{Depth-of-Field Formula}

The ansatz is a similarity relation between the two triangles indicated in the figure (the green triangle has been flipped for clarity). The derivation is carried out for the distance of the near depth-of-field plane $d^{-}$:

$$
\frac{\frac{A}{2}-\frac{h_{o}^{-}}{2}}{\frac{A}{2}}=\frac{A-h_{o}^{-}}{A}=\frac{d^{-}}{d} .
$$

Here, $A$ is the aperture diameter, $h_{o}^{-}$the object side pixel size at the near depth-of-field plane $d^{-}$and $d$ is the object distance that the system is focused at. The object side pixel size is obtained from the (fixed) image-space pixel size $h_{i}$ via the magnification $M^{-}=\frac{f}{d^{-}-f}$ for the near DoF plane:

$$
h_{o}^{-}=\frac{1}{M^{-}} h_{i}=\frac{d^{-}-f}{f} h_{i} .
$$

Inserting Eq. (19) into (18), solving for $d^{-}$and simplifying

$$
d^{-}=\frac{f d\left(A+h_{i}\right)}{A f+d h_{i}}
$$

IThis can be seen by considering an equivalent light field system (LF) to the high-resolution standard system (St): Keeping the field-of-view and the image-side pixel size constant, requires an equivalent focal length of $f / N$. 
is obtained. Similarly, $d^{+}$is derived as

$$
d^{+}=\frac{f d\left(A-h_{i}\right)}{A f-d h_{i}}
$$

Finally, the DoF is given by the difference of these two distances DoF $=d^{+}-d^{-}$, which, after insertion of Eqs. (20) and (21) simplifies to

$$
\operatorname{DoF}=\frac{2 A f d h_{i}(d-f)}{A^{2} f^{2}-d^{2} h_{i}^{2}} .
$$

In the case of $f \ll d$ and $d^{2} h_{i}^{2} \ll A^{2} f^{2}$, which holds for intermediate focal distances for common systems, a useful approximation is obtained:

$$
\mathrm{DoF} \approx \frac{2 d^{2} h_{i}}{A f} .
$$

Applying Eqs. (1) and (2), assuming small angles $\alpha$ for the numerical aperture and re-applying the assumption $f \ll d$, this approximate DoF can be reformulated in terms of the working f-number $\mathrm{F} / \#_{w}$ :

$$
\operatorname{DoF} \approx 2 \mathrm{~F} / \#_{w} h_{i} .
$$

\subsection{Comparison}

We then obtain the following relations for the three comparison systems discussed earlier, Fig. 1. The (LF)

\begin{tabular}{|l|c|c|c|}
\hline property/system & St & LF & LR \\
\hline effective focal length & $f$ & $f / N$ & $f$ \\
effective aperture & $A$ & $A / N$ & $A$ \\
sensor pixel size & $h_{i}$ & $h_{i}$ & $N \cdot h_{i}$ \\
\hline depth of field & $\frac{2 d^{2} h_{i}}{A f}$ & $N^{2} \cdot \frac{2 d^{2} h_{i}}{A f}$ & $N \cdot \frac{2 d^{2} h_{i}}{A f}$ \\
DoF in terms of $\mathrm{F} / \#_{w}$ & $2 \mathrm{~F} / \#_{w} h_{i}$ & $2 N^{2} \mathrm{~F} / \#_{w} h_{i}$ & $2 N \mathrm{~F} / \#_{w} h_{i}$ \\
\hline
\end{tabular}

system has an $N^{2} \times$ larger DoF than the comparison system (St). This effect can, intuitively, be attributed to two factors of $N$ each: 1) the reduced $\mathrm{NA}_{o}^{\mathrm{LF}}$ of the light field subviews, and, 2) the larger object space pixel size $h_{o}^{\mathrm{LF}}$ due to lower resolution, but same field of view as the comparison system (St). The low-resolution standard system (LR) occupies an intermediate position: it has an $N \times$ increased DoF as compared to (St) that is due to effect 2) only. Similar conclusions have been drawn by Levoy et al. ${ }^{8}$

\subsection{Discussion}

This analysis appears to show that the equivalent F/\# for light field systems proposed in Eq. (17) is not an adequate measure for characterizing the DoF. However, the picture is not complete without the digital processing that can be applied to the raw data of light field imaging systems.

In particular, the subviews can be (shifted and) summed to synthesize images that are equivalent to refocused versions of the image that the low-resolution standard system (LR) generates". The effective aperture for the (LF) system in the table becomes equal to the one of the low-resolution standard system (LR).

In order to synthesize the even narrower DoF of the high-resolution standard system (St), digital superresolution techniques ${ }^{9,10}$ must be employed which effectively reduces $h_{i}^{\mathrm{LF}}$ to $h_{i}^{\mathrm{LF}} / N$. In this case, the narrow DoF of the high-resolution standard system (St) can be synthesized fully. In fact, it is even possible to extrapolate synthetic apertures and achieve and even narrower DoF. ${ }^{4}$

Therefore, I propose to interpret the equivalent F/\# introduced in Eq. (17) as a rough measure of the minimum synthetic DoF that can be achieved by a light field imaging system.

\footnotetext{
"Note that the DoF changes with the focal distance; therefore, the digital "sum-and-add" refocusing is not exactly equivalent to the natural one.
} 


\section{CONCLUSIONS}

I have introduced the concept of an equivalent F/\# for light field systems. Just as in standard photography, it is a good measure of light efficiency and SNR in good illumination conditions. As was seen, for low light conditions, light field systems suffer a slight disadvantage that may be exacerbated by registration problems for the then noisy data. In terms of depth-of-field, the equivalent $\mathrm{F} / \#$ provides a rough measure of the minimal synthetic refocus depth-of-field that can be achieved.

\section{ACKNOWLEDGMENTS}

I would like to thank Loïs Mignard-Debise for his careful proof-reading and the valuable suggestions for improvement.

\section{REFERENCES}

[1] Mignard-Debise, L. and Ihrke, I., "A vignetting model for light field cameras with applications to light field microscopy," IEEE Transactions on Computational Imaging (2019).

[2] Mignard-Debise, L., Restrepo, J., and Ihrke, I., "A unifying first-order model for light-field cameras: the equivalent camera array," IEEE Transactions on Computational Imaging 3(4), 798-810 (2017).

[3] Mignard-Debise, L. and Ihrke, I., "Light-field microscopy with a consumer light-field camera," in [2015 International Conference on 3D Vision], 335-343, IEEE (2015).

[4] Manakov, A., Restrepo, J., Klehm, O., Hegedus, R., Eisemann, E., Seidel, H.-P., and Ihrke, I., "A reconfigurable camera add-on for high dynamic range, multispectral, polarization, and light-field imaging," $A C M$ Transactions on Graphics 32(4), 47-1 (2013).

[5] Adelson, E. H. and Wang, J. Y. A., "Single lens stereo with a plenoptic camera," IEEE Transactions on Pattern Analysis $\&$ Machine Intelligence (2), 99-106 (1992).

[6] Ng, R., Levoy, M., Brédif, M., Duval, G., Horowitz, M., Hanrahan, P., et al., "Light field photography with a hand-held plenoptic camera," Computer Science Technical Report CSTR 2(11), 1-11 (2005).

[7] Lumsdaine, A. and Georgiev, T., "The focused plenoptic camera," in [2009 IEEE International Conference on Computational Photography (ICCP)], 1-8, IEEE (2009).

[8] Levoy, M., Ng, R., Adams, A., Footer, M., and Horowitz, M., "Light field microscopy," ACM Transactions on Graphics (TOG) 25(3), 924-934 (2006).

[9] Bishop, T. E., Zanetti, S., and Favaro, P., "Light field superresolution," in [2009 IEEE International Conference on Computational Photography (ICCP)], 1-9, IEEE (2009).

[10] Ihrke, I., Restrepo, J., and Mignard-Debise, L., "Principles of light field imaging: Briefly revisiting 25 years of research," IEEE Signal Processing Magazine 33(5), 59-69 (2016). 